\title{
Determination by Ion Chromatography with Series Bulk Acoustic Wave Detection of Organic Acids in a Chinese Drug Schisandrae Fructus
}

\author{
Xiaorong YAng, Po Chen, Lihua NiE and Shouzhuo YAO ${ }^{\dagger}$ \\ College of Chemistry and Chemical Engineering, Hunan University, Changsha, 410082, P. R. China
}

Keywords Ion chromatography, series acoustic wave detection, organic acid, Schisandrae Fructus

Schisandrae Fructus is a Chinese drug, the dried ripe fruit of Schisandrae chinensis (Turcz.) Bail. or Schisandrae sphenanthera Rehd. et Wils. It acts on the lung, heart and kidney meridians and has the effects of inducing astringency to arrest incessant and excessive loss of body fluid, replenishing qi and promoting the production of body fluid, nourishing the kidney and tranquilizing the mind. ${ }^{1}$ Its physiological activities are related to the presence of organic acids. ${ }^{2}$

In general, organic acids are measured using enzymatic methods or chromatographic techniques. ${ }^{3-7}$ However, enzymatic analyses and gas chromatographic methods have such drawbacks: they are time-consuming or they use large amounts of reagents. Conventional ion chromatography with conductometric detection ${ }^{5}$ is also used to determine organic acids and detects down to about $0.5 \mu \mathrm{g} / \mathrm{ml}$. As such, its sensitivity is not adequate to measure organic acids in certain samples.

In this work, a new type detection system for IC called a series bulk acoustic wave (SBAW) ${ }^{8,9}$ was used to develop a procedure for the determination of organic acids in Schisandrae Fructus, when incorporated into a non-suppressed anion exchange chromatographic system. It was more sensitive than IC with conductometric detection.

\section{Experimental}

\section{Reagents and materials}

Organic acids (malic, citric and tartaric) were purchased from Beijing Chemicals Co. (Beijing, China) as analytical grade. All other chemicals were of analytical reagent grade and were used as received. Deionized water was used for preparation of the eluent and standard solutions. The eluent, standard and sample solutions were filtered through a $0.45 \mu \mathrm{m}$ membrane (Millipore, USA).

\section{IC apparatus}

IC was equipped with an autoinjector (SIL-6B), a liq-

\footnotetext{
$\doteqdot$ To whom correspondence should be addressed.
}

uid delivery pump (LP-6A), a CTO-6AS column oven and a system controller. The analytical column was a Shimpack IC-A1 $(4.6 \mathrm{~mm}$ i.d. $\times 100 \mathrm{~mm} \mathrm{~L})$, filled with $12.5 \mu \mathrm{m}$ polymethacrylate substrate incorporating a quaternary base as a functional group. A Shim-pack IC-GA1 guard column $(4.6 \mathrm{~mm}$ i.d. $\times 10 \mathrm{~mm} L)$ preceded the analytical column. All this equipment above was purchased from Shimadzu (Kyoto, Japan). The detection system consisted of the SBAW detector and an $\mathrm{F} / \mathrm{V}$ converter.

\section{IC conditions}

The eluent was $1.5 \mathrm{mM}$ phthalic acid with $\mathrm{pH} 4.0$ used at a flow rate of $1.2 \mathrm{ml} / \mathrm{min}$ and the injection volume was $20 \mu \mathrm{l}$. The temperature of column and detector was set at $38^{\circ} \mathrm{C}$.

\section{SBAW detector}

The SBAW detector was composed of a $9 \mathrm{MHz}$ ATcut quartz crystal oscillator connected in series and a conductivity detection cell. The detection cell was made as follows: two $1 \mathrm{~mm}$ platinum wires, as conductivity electrodes, were inserted oppositely in the detection cell, with a distance of $0.5 \mathrm{~mm}$ between them. The body of the detector was made of two plexiglass plates with a thickness of $5 \mathrm{~mm}$, within which a cylindrical trough ( $2 \mathrm{~mm}$ in diameter and $8 \mathrm{~mm}$ in length) was used as detection cell. Two $0.5 \mathrm{~mm}$ stainless-steel tubes were connected oppositely to the detection cell and used as inlet and outlet for the mobile phase, separated by $7 \mathrm{~mm}$. One of the platinum electrodes was connected to the quartz crystal; together with another platinum wire, it served as the feedback network of the TTL-IC oscillator. The frequency signal from the detector was sent either to the digital counter to give direct frequency recording or to the F/V conversion circuit connected to the chromatography workstation to get voltage information. The base noise and hence the sensitivity of the SBAW is influenced by temperature. When the SBAW detector temperature is equal to that of the column, the lowest noise and drift levels are obtained. Hence, the temperature of the SBAW detector is kept the same as that of the column, $38^{\circ} \mathrm{C}$. 


\section{F/V converter}

A universal frequency counter (Model SC-7201, Iwatsu, Japan) was used to investigate the performance of the BAW sensor with respect to frequency shift. As the frequency data must be transformed into a format which can be used by the chromatographic workstation, a frequency-to-voltage converter (made in our laboratory) was used to transform the frequency signal of the BAW detector to a CR-4A Chromatopac data processor (Shimadzu, Japan), which is used to record chromatograms in real time and to integrate peak areas.

\section{Results and Discussion}

\section{Choice of the eluent}

The sensitivity of an SBAW detector is mostly affected by the eluent conductivity $\left(G_{0}\right)$, when all design parameters of the detector are kept unchanged. ${ }^{8,9}$ In order to achieve a sensitive response, various dilutions of phthalic acid solution with $\mathrm{pH} 4.0$ were evaluated (Fig. 1). From Fig. 1, the detector sensitivity increases

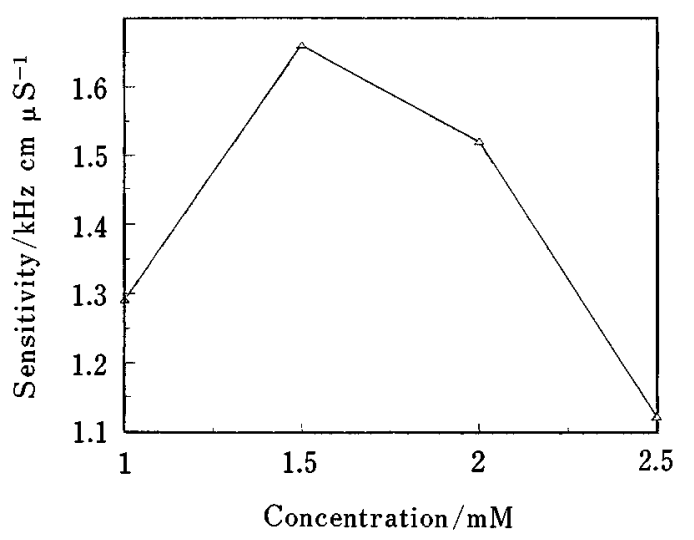

Fig. 1 Effect of eluent concentration on detector response sensitivity. The signal to noise ratio is 3 .

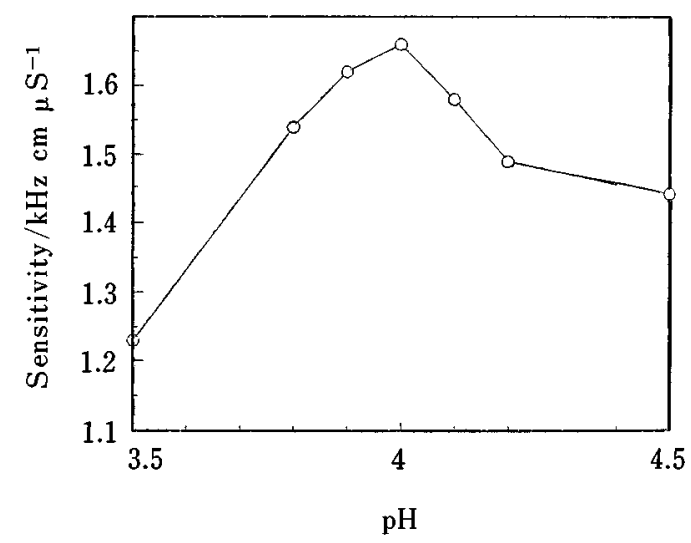

Fig. 2 Effect of eluent $\mathrm{pH}$ on detector response sensitivity. The signal to noise ratio is 3 . with increasing the concentration of the eluent until a maximum is reached, and then it decreases as the eluent concentration increases further. The maximum sensitivity is reached at $1.5 \mathrm{mM}$ phthalic acid solution with $\mathrm{pH}$ 4.0. Also, the conductivity of the eluent can also be affected by its $\mathrm{pH}$ and thereby the detector sensitivity is changed. To seek a suitable $\mathrm{pH}$, the eluent $\mathrm{pH}$ was changed; the obtained results are shown in Fig. 2. As can be seen in Fig. 2, pH 4.0 of the eluent with $1.5 \mathrm{mM}$ gave the best sensitivity.

In this work, $1.5 \mathrm{mM}$ phthalic acid solution with $\mathrm{pH}$ 4.0 was used as the eluent, since it gave not only the best sensitivity but also a reasonable retention time. Figure 3 shows a typical chromatogram obtained for $1.5 \mathrm{mM}$ phthalic acid solution with $\mathrm{pH} 4.0$ as the eluent, where the organic acids tested peaks were well resolved.

\section{Linear range, recovery and precision}

The linear range of the proposed method was investigated by analyzing standard solutions containing a mixture of three analytes with known concentrations ranging from $0.20-400 \mu \mathrm{g} / \mathrm{ml}$. Their linear range and correlation coefficients are given in Table 1.

To evaluate the accuracy of the proposed IC-SBAW method, under the optimized chromatographic and detector conditions described in the Experimental section, recovery experiments were performed by spiking real samples with aliquots of standard organic acid

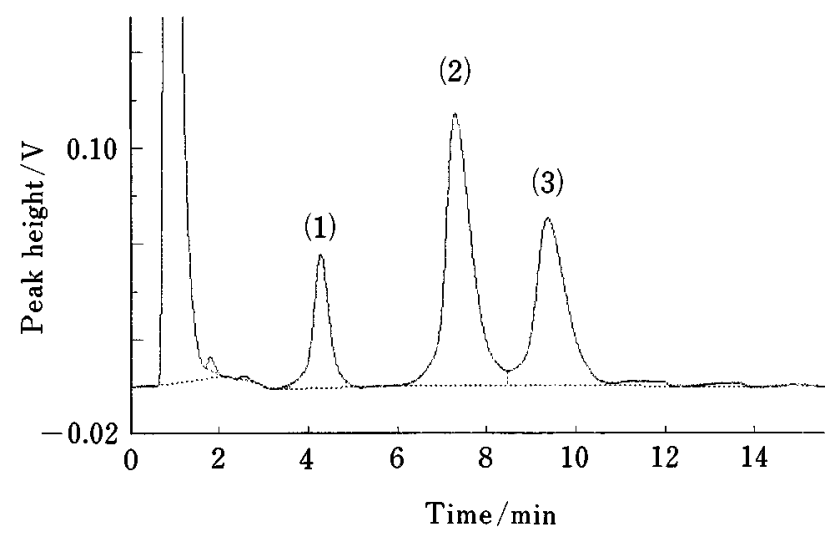

Fig. 3 Chromatogram of a sample: (1) malic acid (7.04 $\mu \mathrm{g} / \mathrm{ml}),(2)$ citric acid $(17.42 \mu \mathrm{g} / \mathrm{ml})$, (3) tartaric acid (4.40 $\mu \mathrm{g} / \mathrm{ml})$.

Table 1 Linear range and detector limit of organic acids

\begin{tabular}{lccc}
\hline \multicolumn{1}{c}{ Acid } & $\begin{array}{c}\text { Detection limit/ } \\
\mu \mathrm{g} \mathrm{ml}^{-1}\end{array}$ & $\begin{array}{c}\text { Linear range/ } \\
\mu \mathrm{g} \mathrm{ml}^{-1}\end{array}$ & $\begin{array}{c}\text { Correlation coefficient } \\
(n=7)\end{array}$ \\
\hline Malic acid & 0.036 & $0.045-45$ & 0.9974 \\
Citric acid & 0.065 & $0.070-70$ & 0.9992 \\
Tartaric acid & 0.041 & $0.050-50$ & 0.9989 \\
\hline
\end{tabular}


solutions. The obtained recoveries $(n=7)$ were 95 $107 \%$.

Precision was tested by performing seven analyses of a standard mixture containing $10 \mathrm{ppm}$ malic, $20 \mathrm{ppm}$ citric and $10 \mathrm{ppm}$ tartaric acids. The relative standard deviations $(n=7)$ of malic, citric and tartaric acid were $2.1,1.5$ and $1.9 \%$, respectively.

\section{Analysis of Schisandrae Fructus}

Each powdered sample $(1 \mathrm{~g})$ was dissolved in $\mathrm{H}_{2} \mathrm{O}$ and the solution was extracted with acidified ethanol. The extract was evaporated and the $\mathrm{pH}$ of the residue was adjusted to $7.5-8.5$ with $10 \% \mathrm{Na}_{2} \mathrm{CO}_{3}$ solution and treated with ethyl acetate to remove tannins, gallic acid and other impurities. A portion $(20 \mu \mathrm{l})$ of the resulting solution was analyzed by IC-SBAW.

The proposed IC-SBAW was applied to determine levels of organic acids in the samples. The concentrations of malic, citric and tartaric acid in Shisandrae Fructus were 352,871 and $220 \mu \mathrm{g} / \mathrm{g}$ and the standard deviations $(n=7)$ of malic, citric and tartaric acid were 11,26 and 6 , respectively.

\section{Comparison of SBAW detector with conductometric detector for IC}

Compared with conventional conductometric detection for IC, SBAW detection is more sensitive and gives more stable baselines, so it can avoid such phenomena as double layer capacitance formation or Faradaic impedance, which affect the sensitivity and accuracy of determination of ions. Under the optimum conditions, the detection limits of the IC-SBAW method, defined as a signal-to-noise ratio of 3 , were $0.036,0.065$ and $0.041 \mu \mathrm{g} / \mathrm{ml}$ to malic, citric and tartaric acid, respectively (Table 1), whereas those of IC with conductometric detection were $0.52,0.86$ and 0.53 $\mu \mathrm{g} / \mathrm{ml} .^{5}$

The ion chromatographic method using an SBAW detector is advantageous in its sensitivity. It has successfully been applied to the determination of malic, citric and tartaric acid in Schisandrae Fructus.

\section{References}

1. F. Wang and J. Luo (ed.), "A Practical Chinese-English Dictionary of Traditional Chinese Materia", p.54, Hunan Science \& Technology Press, Changsha, 1994.

2. Q. Lin (ed.), "Analytical Chemistry of the Natural Medicinal Material", p. 143, Science Press, Beijing, 1977.

3. J. D. Blake and M. L. Clarke, J. Chromatogr., 398, 265 (1987).

4. S. Peldszus, P. M. Huck and S. A. Andrews, J. Chromatogr. A, 723, 27 (1996).

5. Z. Gao and C. Fu, Fenxi Ceshi Xuebao, 13, 32 (1994).

6. J. E. Lawrence and P. Koutrakis, Environ. Sci. Technol., 28, 957 (1994).

7. J. S. Fritz, J. Chromatogr., 546, 111 (1991).

8. S. Yao, B. Yu, P. Chen, L. Nie, M. Yang and W. Zhu, Instrum. Sci. Technol., 24, 247 (1996).

9. P. Chen, L. Nie and S. Yao, J. Chromatogr. Sci., 33, 268 (1995).

(Received June 4, 1997)

(Accepted November 17, 1997) 